\title{
Logic Puzzles as Mental Gymnastics for LSAT Preparation
}

\author{
Marina Karapetyan \\ Yerevan State University
}

$\mathrm{I}$

$\mathrm{n}$ recent years a new standardized test has been administered in Armenia to evaluate law school applicants. The LSAT (Law School Admission Test) has been used in the USA, Canada and Australia since 1991. It tests logical and verbal reasoning, as well as reading comprehension skills. It is designed especially to measure a candidate's ability to think logically and draw logical conclusions from facts and premises. The test consists of several sections, of which the Analytical and Logical Reasoning sections seem most unfamiliar to Armenian students and hence frightening.

This research is aimed at exploring amusing and learner-friendly ways of fostering critical thinking skills and attention to detail as basic skills for the Analytical Reasoning questions of the LSAT. The method suggested here is through logic games, with a focus on their specific type commonly referred to as logic grid puzzles. For one thing, this logic game is perceived as an enjoyable leisure activity, a kind of mental gymnastics, a warm-up in terms of both English proficiency and principles of reasoning and drawing conclusions. Moreover, it ranges from easy to more challenging types, which can provide a good basis for beginners.

What is a logic puzzle and what purpose does it serve? A logic puzzle can be defined as a riddle that requires deductive reasoning. To put it simply, it is a test of our ability to think clearly. A typical puzzle has a short introduction, which sets the situation and presents the problem as well as provides some clues to help deduce the solution. For example, you might be told that four people each built a house in a different part of a town in a different month of the year, and the task would be to match up each person's name with the type of housing, the part of the town and the month when the house was built. Each option is used only once. Each puzzle has only one possible solution and can be solved using simple logical processes.

However, few people can solve logic puzzles "in mind"; most people record the information deduced in the so-called solving grid, which helps them clearly see the situation and make further deductions. The grid allows you to cross-reference every possible option in the scenario. There are two kinds of grids: the "crosshatch" grid and the "fillin" grid. Which is more useful depends on each particular puzzle. Very often, both can be effective. Here's the crosshatch grid with a very simple puzzle scenario: ${ }^{1}$ Four babies were all born within an hour of one another at our local hospital. Each baby was born at a different time (7:00, 7:15, 7:30, and 7:45) that evening, and the ward supervisor came around the next day to take a snapshot of each of the happy mothers (Dot, Janis, Serena, and Vicky) with her newborn (two boys named Aidan and Travis, and two girls named Colleen and Reba). 


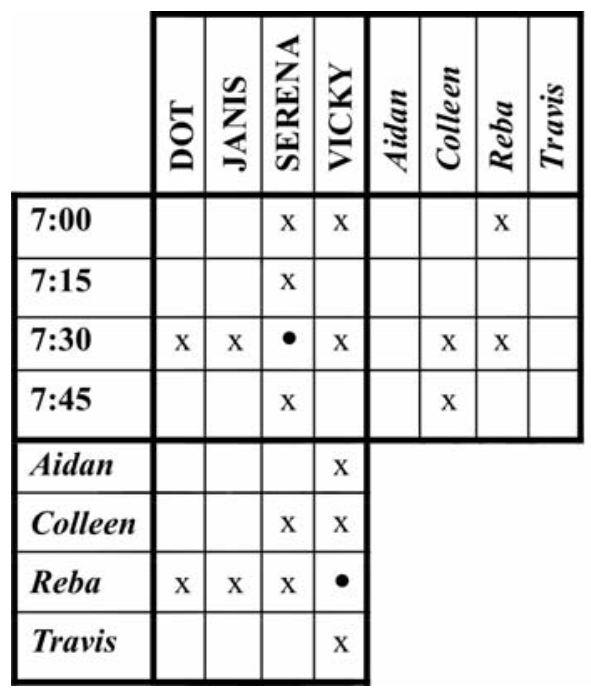

Source: Dell Logic Puzzles, 2003: 6

You fill in all the squares in the grid. True assertions can be represented by a dot, while false assertions can be represented by an X. If you know from the clues, for example, that Serena's son was born at 7:30, you can add a dot in the box where the Serena column and 7:30 row meet. Similarly, if the clues tell you that Vicky gave birth to Reba at some point after Colleen was born, you can cross out both the options that Reba was the first child to be born that evening and that Colleen was the last, and consequently, the option that Vicky gave birth to Reba at 7:00. You continue doing this for every clue until you have filled the board with enough $X$ 's and O's to make a final deduction. The logic is this: if $\mathrm{A}=\mathrm{B}$, and $\mathrm{B}=\mathrm{C}$, then $\mathrm{A}$ must equal $\mathrm{C}$. In the same way, if $\mathrm{A}=\mathrm{B}$, and $\mathrm{B}=/=\mathrm{D}$, then A cannot equal D. That is, if Vicky $=$ Reba and if Reba $=/=7: 00$, then Vicky $=/=7: 00$ (although this option was automatically eliminated when we marked the hour Serena's baby was born.

Below is a sample fill-in grid:

\begin{tabular}{|c|c|c|}
\hline Dot & & \\
\hline Janis & & \\
\hline Serena & & $7: 30$ \\
\hline Vicky & Reba & \\
\hline
\end{tabular}

As can be seen, the crosshatch grid gives more opportunity for eliminating possibilities; however, the fill-in grid can be useful for solving bigger and more challenging puzzles when most deductions have already been recorded and the puzzler needs to arrange conclusions in a more orderly manner.

The process of deductive reasoning can be rather complex. Conclusions are obtained step by step from each clue, with each step leading to a new claim. The claims are the result of an application of valid arguments to the clues or to previously formed claims. That is, each new conclusion becomes a kind of premise for further conclusions. Thus the clues (or premises) can be considered as a basis for making deductions. If you understand the clues clearly and follow them, you should make valid arguments, and, consequently, valid conclusions.

The first thing to do in solving a logic grid puzzle is to read the introduction and clues carefully, and make sure you understand what you are to figure out. The main skills required for solving small logic puzzles consist of natural language understanding and simple logical reasoning. Quite often, some of the clues can be contained in the introduction. For example, the introduction to the puzzle about the newborn babies above might tell you that of the four babies, two are girls (named Colleen and Reba) and two are boys 
(Aidan and Travis). This valuable information rules out Colleen and Reba as Serena's baby, as Clue 1 says: "Serena's son was born at 7:30". It also becomes obvious that neither of the girls was born at 7:30.

Let us now explore the structure and nature of the LSAT Analytical Reasoning section and see how logic grid puzzles are related to it and how they could help foster the skills required for this section.

The purpose of the Analytical Reasoning section is to establish how well a law school applicant understands complex relationships between a wide range of objects, places, people or events, and demonstrates the ability to combine them to make valid deductions. This section contains four problems, or games, with a group of four to eight questions related to each game. A game has an introduction, which tells you what is involved and what must be done (for instance, Clarence is organizing his file drawers. He wants to put all of his notes from five economics classes - A, B, C, D, E-in the chronological order in which he took the classes), and several rules or conditions to govern the game. The rules are mainly similar to the clues in a logic grid puzzle. Some are straightforward, others are somewhat ambiguous and it is only possible to make a deduction by combining two or more rules. In addition, there is a type of rules specific to the LSAT - the socalled hypothetical rules that are often merged with the questions. Such rules are worded as "if-then" conditionals. For example: If class $A$ was the fourth class that Clarence took (antecedent), then which one of the following statements must be true (consequent)? The following is an example of a more complex hypothetical rule: If Clarence took class $C$ first, and class $E$ third, which class did he take fourth? As can be seen, the rule contains more than just one premise. A hypothetical rule only applies to the question in which it appears. The other questions in the group are not affected.

In fact, the setups and tasks for logic grid puzzles and the LSAT games are very much alike. The former usually introduce a scenario and then ask to make certain deductions based on a list of clues. Similarly, the latter have an introduction, a set of rules to help figure things out and individual questions related to the setup. Both assignments are based on deductive guesses and can be worked out by using a solving grid to record inferences.

Although the principles of solving the two types of activities are similar, the questions in the LSAT games are more precise than the ones in logic grid puzzles. While a logic puzzle may ask you simply to match, group or put things in order, an analytical game may tell you to identify an acceptable order from among multiple choice answers, or to select the true or the false option, or to give a complete and accurate list of something, etc.

In both activities, the rules (clues) are the reasons or justifications for a claim or a conclusion. There is a subtle difference, however. In the LSAT, and in logic science at large, one premise supplements another to result in a valid conclusion. On the contrary, in a logic grid puzzle, each clue can be an independent premise that supports a conclusion, though sometimes you need to look at two clues collectively to see what can be ruled out and what belongs together.

As was already stated, the principal skills required for both types of activities are 
sequencing, grouping and matching sets of things, or a combination of these skills. Another common skill is sketching, that is the ability to outline or summarize the main ideas to assist in reaching a solution. Sketching proves especially useful in the conditions of time pressure. While it is not the case with logic grid puzzles, which you do for fun at leisure, time pressure is always an issue on the test (less than 10 minutes can be spent on each problem).

The above mentioned solving grid is only one kind of sketching, but one can work out simpler and clearer sketches as well, just to help keep the initial data and new deductions in mind and thus handle a considerable amount of information. On the LSAT games, you may even be urged to make individual diagrams for each question because of the hypothetical form of the questions. In such a case, you need a less time-consuming way to organize information. The following is an example of an easy diagram telling there are five products $(\mathrm{P})$ to be presented by a different one of five salespeople $\mathrm{S}$ and that two of the presentations should take place on Monday, one on Tuesday, and the last two on Wednesday. ${ }^{2}$

$$
\begin{array}{lll}
\text { Mon } & \text { Tues } & \text { Wed } \\
\text { S1 : P1 } & \text { S3:P3 } & \text { S4:P } 4 \\
\text { S2 : P2 } & & \text { S5: P5 }
\end{array}
$$

The above description of the LSAT games and logic grid puzzles confirms the idea that the two kinds of activities differ in some points and should not be equated. Although there is a lot in common between the two, as we stated before, it is necessary to keep in mind that logic puzzles cannot be the only activity to prepare for the Analytical Reasoning section of the LSAT. They can only be an excellent springboard for proceeding to the LSAT games. In fact, they give test-takers a solid intuitive grasp of the logical structure and develop various reasoning skills, including an ability to identify the most basic parts of an argument - premises and conclusions, an ability to perform a critical analysis, as well as comprehend, evaluate and complete a range of arguments, an ability to identify and match patterns of reasoning and to recognize conclusions that depend on unsupported assumptions, etc. These vital skills will serve as a basis for a further and more comprehensive study of logical relationships and will contribute to success in the Logical Reasoning section of the LSAT.

To sum up, we recommend logic grid puzzles to students of English and law-school candidates as a preliminary and a supplement to the actual LSAT preparation for the following reasons:

- they are viewed as leisure activities and hence can be solved for pleasure and arouse interest and motivation for further LSAT preparation;

- they do not press for time and can be done at a relaxed pace;

- they range from easy to difficult, which allows step-by-step progress;

- they provide a warm-up not only on the principles of reasoning, but also in the English language proficiency and thus can be used by students whose knowledge of English is not sufficient for the actual LSAT preparation. 
In addition, logic puzzles develop applicants' logic at large, which builds a solid background for success in other parts of the test.

\section{Notes:}

1. The puzzles are borrowed from $<$ http://www.testsherpa.com/lsat/>.

2. The source of the diagram is The Official LSAT SuperPrep 2007:144.

\section{References:}

1. Dell Logic Puzzles. (June 2003) No 83. Dell Magazines, Norwalk.

2. The Official LSAT SuperPrep. (2007) Law School Admission Council. Newtown, PA.

3. <http://www.logic-puzzles.org/>

4. <http://en.wikipedia.org/wiki/Logic_puzzle>

5. $<\mathrm{http}: / /$ www.testsherpa.com/lsat/>

6. $<\mathrm{http}: / /$ www.lsattestquestions.com/>

\section{Spuúupufiul|ufi q[nı|ulnnunnıl|itipn

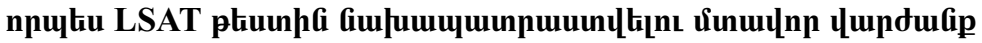

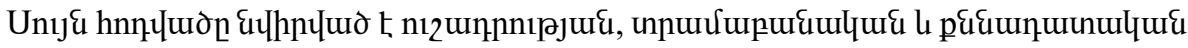

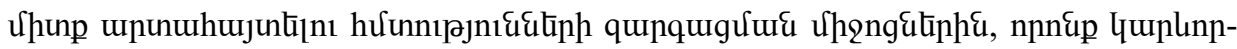

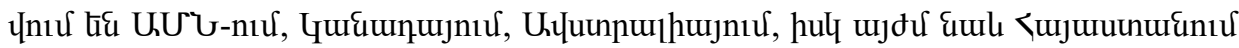

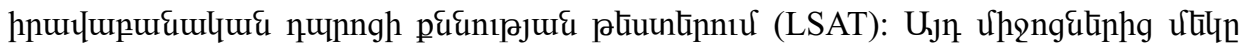

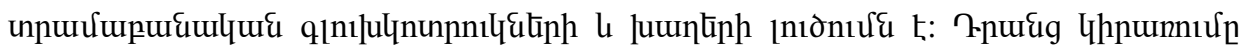

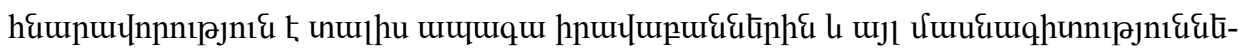

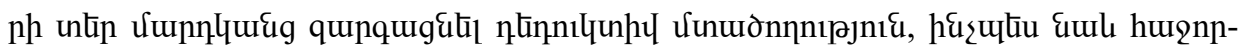

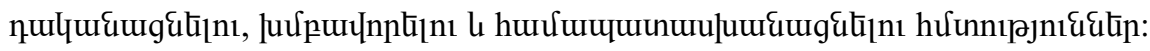

